\title{
TECCIENCIA
}

\section{Origin of the Milky Way Disk of Satellites: Collision of Two Disk Galaxies}

\author{
El Origen del Disco de Satélites de la Vía Láctea: Colisión de Dos Galaxias Disco
}

\author{
Omar Alfonso Bohórquez Pacheco ${ }^{1 *}$, Rigoberto A. Casas Miranda ${ }^{1}$

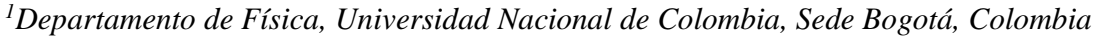

\begin{abstract}
The dwarf satellite galaxies of the Milky Way are structures that are distributed around the galaxy in an anisotropic manner on a structure called Disk of Satellites (DoS). This structure does not match the predictions made by the cold dark matter cosmological model, which predicts a fully isotropic distribution around the Milky Way. To explain the spatial distribution of the satellite galaxies several models have been proposed. One of these models raises the possibility that these galaxies and their spatial distribution were generated by the collision of two disk galaxies several billion years ago. This paper shows N-body simulations performed with the Gadget-2 code to determine whether this event occurred in this way. An analysis for different mass ratios between the incident galaxy, called ghost galaxy, and the host galaxy or target galaxy is performed. Finally a cluster analysis on the collision remains is performed to determine the spatial distribution of the satellite galaxies.
\end{abstract}

Keywords: Local Group Dwarf Galaxy, Galaxy disk, Disk of Satellites, Dark Matter.

\section{Resumen}

Las galaxias enanas satélite de la Vía Láctea son estructuras que están distribuidas alrededor de la Galaxia de manera anisotrópica, formando una estructura conocida como el Disco de Satélites. Esta estructura no coincide con las predicciones hechas por el modelo cosmológico de materia oscura fría, el cual predice una distribución totalmente isotrópica alrededor de la Vía Láctea. Varios modelos han sido propuestos para para explicar la distribución de las galaxias satélite. Uno de estos modelos propone la posibilidad de que estas galaxias y su distribución espacial fueron generados en la colisión de dos galaxias disco hace varios miles de millones de años. En este trabajo se presentan simulaciones de $\mathrm{N}$ cuerpos realizadas con el código Gadget-2 para determinar si este evento ocurrió de esta manera. Se hace un análisis para distintas razones de masa entre la galaxia incidente, llamada también galaxia fantasma y la Vía Láctea. Finalmente, presentamos un análisis de agrupamiento sobre los restos de la colisión para determinar la distribución espacial de las galaxias satélite.

Palabras clave: Galaxias Enanas del Grupo Local, Disco galáctico, Disco de Satélites, Materia Oscura.

\section{Introduction}

The study of dwarf galaxies is important because of their high content of old, low-metallicity stars, which can give information about properties of the universe in its infancy.

*Corresponding Author.

E-mail: oabohorquezp@unal.edu.co
Furthermore, the distribution of satellite galaxies of the Milky Way (which because of their small size are classified as dwarf galaxies) contradicts the predictions made by the cosmological model of cold dark matter (CDM).
How to cite: Bohórquez Pacheco, O. A., Casas Miranda, R. A., Origin of the Milky Way Disk of Satellites: Collision of Two Disk Galaxies, TECCIENCIA, Vol. 12 No. 21, 33-38, 2016, DOI: http:/dx.doi.org/ 


\section{TECCIENCIA}

This theory predicts that the distribution of substructures within dark matter halos like the Milky Way should be isotropic, which does not match the observations [1], showing that the distribution of these objects is completely anisotropic.

Most of the satellite galaxies of the Milky Way are distributed in a plane perpendicular to the plane of the galaxy, which on average is inclined 88 degrees with respect to the disc of the galaxy [3]; this plane is known as satellite disk (DOS). It has not been possible find a model that takes into account the amount and spatial distribution of these galaxies. There have been several proposed solutions, one of which suggests that these satellites originated a collision of the Milky Way with another disk galaxy billions of years ago [2].

This paper describes several numerical N-body simulations of collisions between the Milky Way galaxy and the hypothetical disk galaxy (also known as Ghost Galaxy) that might have given rise to the disk of Milky Way satellite galaxies. The name "Ghost Galaxy" relates to the idea of a "ghost car", when a vehicle runs over a person but nobody really knows which car perpetrated the act.

The paper is organized as follows: in Section 2 the theoretical elements underlying our model (density profiles, orbit pre-collision and simulation initial conditions) are defined; in Section 3 analysis of the results and the corresponding discussion is made, and finally the conclusions are presented in Section 4.

\section{Methods}

In the proposed model, billions of years ago the Milky Way interacted with a disk galaxy (Ghost Galaxy), whose mass is considered to be much less than that of the Milky Way. This interaction generated tidal tails that are regarded as possible progenitors of satellite galaxies of the Milky Way [2].

\subsection{Density profiles}

To recreate the structure of disk galaxies like the halo of dark matter, the disc and bulge the following density profiles were used.

\subsubsection{Hernquist Profile (Halo and Central bulge)}

$\rho=\frac{M a}{2 \pi r(a+r)^{2}}$,

Here $M$ represents the mass of the Halo or bulge, $r$ is the point where the potential is measured and $a$ is the radius associated with the spheroid's core (radial scale length) [4].

\subsubsection{Exponential Profile (Disk)}

$\rho(r, z)=\frac{M_{d}}{2 \pi R_{d}} e^{-\frac{R}{R_{d}}} \operatorname{sech}^{2} \frac{z}{z_{0}}$

Here $M_{d}$ is the mass of the disk, $R_{d}$ is the scale length and $z_{0}$ is the disc thickness. [5]

\subsection{Pre-Collision Orbit}

For the collision a prograde orbit for the satellite is chosen (see Fig. 1). These orbits generate prominent tidal tails [6]. The kinematic equations of motion are based on the models by Van de Kamp [7].

$$
\begin{aligned}
& v_{r}=\sqrt{\frac{\mu}{p}} \operatorname{esen}(\theta) \\
& v_{\theta}=\sqrt{\frac{\mu}{p}}(1+e \cos (\theta))
\end{aligned}
$$

Here $r$ is the distance of the ghost galaxy to the Milky Way (the Milky Way being located at the focus of the orbit), $p$ is the distance of closest approach (impact parameter), $e$ is the eccentricity of the orbit, $\theta$ is the angle of radius vector with respect to the $x$ axis, and $v_{r}, v_{\theta}$ are the parallel and perpendicular components of the velocity with respect to the radial position vector.

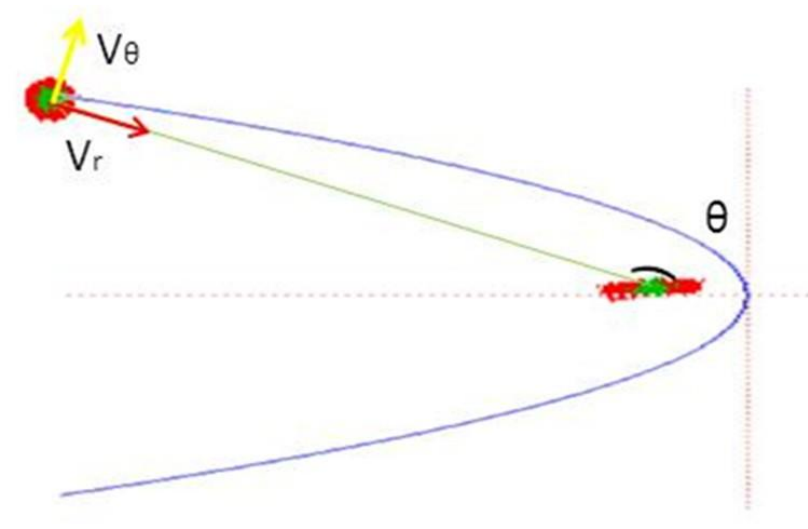

Figure 1 Parabolic prograde orbits [5].

\subsection{Initial conditions for the simulations}

Simulations were performed for mass ratios between the Milky Way and the ghost galaxy of 4, 8, 12 and $16 \%$. Tables 1-3 summarize the parameters used in different simulations. 


\section{TECCIENCIA}

Table 1 Parameters for the Milky Way [8] [9].

\begin{tabular}{cccc}
\hline Parameter & Description & Milky Way & Units \\
\hline $\mathbf{M}_{\mathrm{h}}$ & Mass dark matter halo & $1,55 \times 10^{12}$ & $\mathrm{M}_{\odot}$ \\
$\mathbf{R}_{\mathrm{vir}} \mathbf{0} \mathbf{R}_{200}$ & Virial radius of the halo & 244.8 & $\mathrm{kpc}$ \\
$\mathbf{a}_{\mathrm{H}}$ & Halo scale length & 37 & $\mathrm{kpc}$ \\
$\mathbf{M}_{\mathrm{d}}$ & Disk mass & $5,0 \times 10^{10}$ & $\mathrm{M}_{\odot}$ \\
$\mathbf{R}_{\mathrm{S}}$ & Radial scale length of the disc & 4 & $\mathrm{kpc}$ \\
$\mathbf{R}_{\mathrm{Z}}$ & Vertical scale length of the disk & 0.3 & $\mathrm{kpc}$ \\
$\mathbf{M}_{\mathrm{b}}$ & Mass bulge & $1.8 \times 10^{10}$ & $\mathrm{M}_{\odot}$ \\
$\mathbf{R}_{\mathrm{SB}}$ & Bulge scale length & 0.6 & $\mathrm{kpc}$ \\
$\mathbf{C}$ & Concentration parameter & 12 & - \\
\hline
\end{tabular}

Table 2 Parameters of different ghost galaxies used in this study.

\begin{tabular}{ccccc}
\hline Parameter & Description & $\mathbf{1 2 \%}$ & $\mathbf{1 6 \%}$ & Units \\
\hline $\mathbf{M}_{\mathbf{h}}$ & Mass dark matter halo & $1,94 \times 10^{11}$ & $2,48 \times 10^{11}$ & $\mathrm{M}_{\odot}$ \\
$\mathbf{R}_{\mathbf{v i r}} \mathbf{0} \mathbf{R}_{\mathbf{2 0 0}}$ & Virial radius of the halo & 122,4 & 132,9 & $\mathrm{kpc}$ \\
$\mathbf{a}_{\mathbf{H}}$ & Halo scale length & 11,7 & 12,7 & $\mathrm{kpc}$ \\
$\mathbf{M}_{\mathbf{d}}$ & Disk mass & $6,25 \times 10^{9}$ & $8 \times 10^{9}$ & $\mathrm{M}_{\odot}$ \\
$\mathbf{R}_{\mathbf{S}}$ & Radial scale length of the disc & 1,41 & 1,60 & $\mathrm{kpc}$ \\
$\mathbf{R}_{\mathbf{Z}}$ & Vertical scale length of the disk & 0,106 & 0,120 & $\mathrm{kpc}$ \\
$\mathbf{M}_{\mathbf{b}}$ & Mass bulge & $2,25 \times 10^{9}$ & $2,88 \times 10^{9}$ & $\mathrm{M}_{\odot}$ \\
$\mathbf{R}_{\mathrm{SB}}$ & Bulge scale length & 0,30 & 0,33 & $\mathrm{kpc}$ \\
$\mathbf{C}$ & Concentration parameter & 21.8 & 21.8 & - \\
\hline
\end{tabular}

Table 3 Parameters of the different simulated parabolic orbits.

\begin{tabular}{ccccc}
\hline Parameter & Description & $\mathbf{1 2 \%}$ & $\mathbf{1 6 \%}$ & Units \\
\hline $\mathbf{P}$ & Impact parameter & 45 & 45 & $\mathrm{kpc}$ \\
$\boldsymbol{\mu}$ & Gravity parameter & $2,41 \times 10^{32}$ & $2,49 \times 10^{32}$ & $\mathrm{~kg} / \mathrm{Nm}^{2}$ \\
$\boldsymbol{\Theta}$ & Angle measured with respect to x & 130 & 130 & $\circ$ \\
$\mathbf{X}$ & Initial position in x & 0 & 0 & $\mathrm{kpc}$ \\
$\mathbf{Y}$ & Initial position in y & $-80,98$ & -80.98 & $\mathrm{kpc}$ \\
$\mathbf{Z}$ & Initial position in z & 96,50 & 96.50 & $\mathrm{kpc}$ \\
$\mathbf{v}_{\mathbf{x}}$ & Initial velocity in x & 0 & 0 & $\mathrm{~km} / \mathrm{s}$ \\
$\mathbf{v}_{\mathbf{y}}$ & Initial velocity in y & 319,60 & 324,54 & $\mathrm{~km} / \mathrm{s}$ \\
$\mathbf{v}_{\mathbf{z}}$ & Initial velocity in z & $-149,03$ & -151.33 & $\mathrm{~km} / \mathrm{s}$ \\
\hline
\end{tabular}

\section{Results and Discussion}

Here we present the results of the simulated collisions. Figure 2 shows that for a $12 \%$ and $16 \%$ mass ratio, after the collision a disk-like structure forms at a $90^{\circ}$ angle with respect to the galactic plane. However, for the $16 \%$ mass ratio scenario the model Milky Way is heated by the collision and completely loses its disk structure. This situation is not compatible with observations.

It should be noted that simulations corresponding to mass ratios of $4 \%$ and $8 \%$ respectively are not presented in this document since for those cases, interactions between galaxies can last for longer than a Hubble time. 


\section{TECCIENCIA}
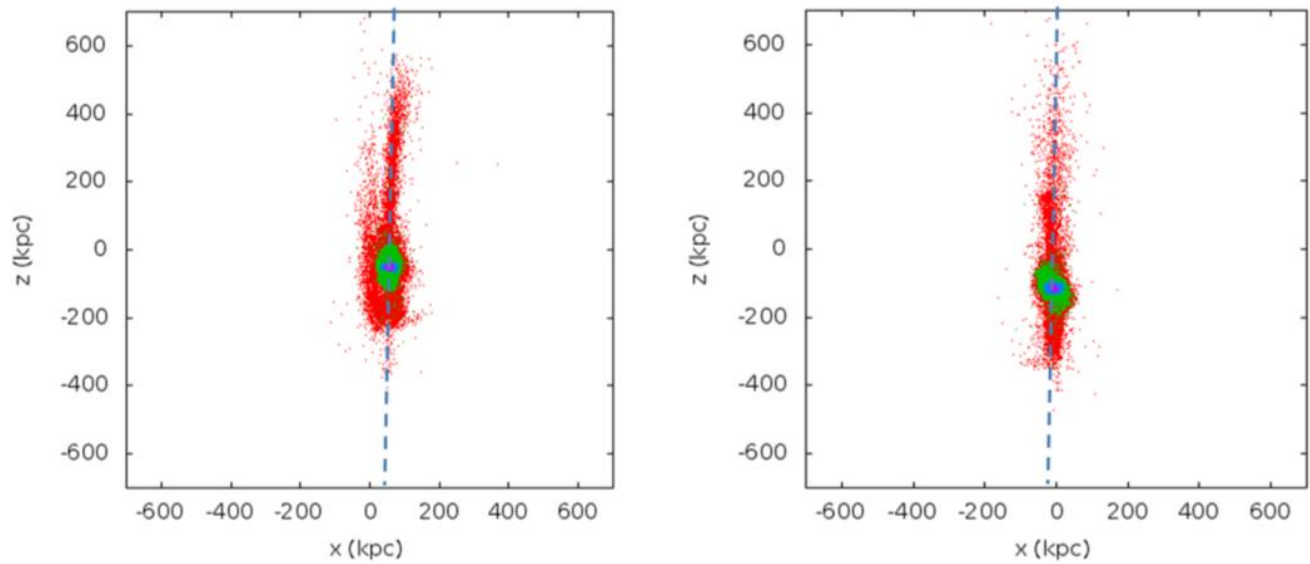

Figure 2 Results of the simulated collision between the Milky Way (green dots) and a Ghost Galaxy (red dots) after 10 Gyr for a $12 \%$ (left) and a $16 \%$ (right) mass ratio.
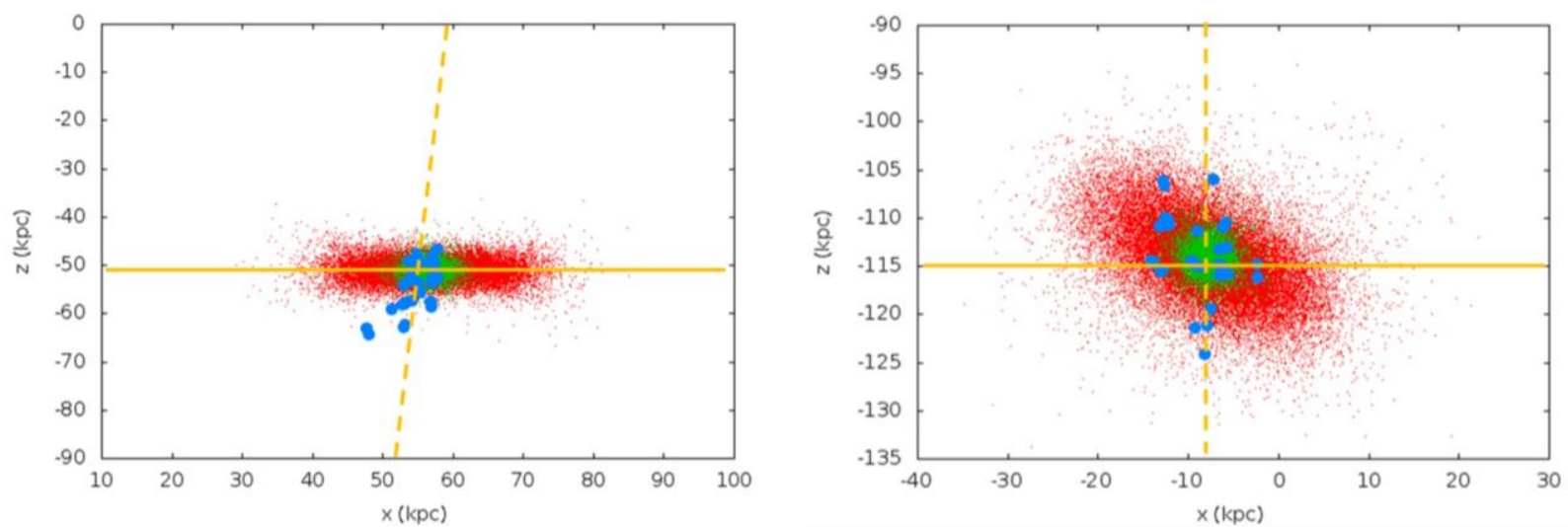

Figure 3 Zoomed-in view of the simulated collision between the Milky Way (green dots) and a Ghost Galaxy (red dots) after $10 \mathrm{Gyr}$ for a $12 \%$ (left) and a $16 \%$ (right) mass ratio. The blue dots show the location of plausible stellar groups as estimated by the friends-of-friends code. The groups were found using a linking length value of $1 \mathrm{kpc}$.

The FoF (friends-of-friends) code was used to search for groups that might be the dwarf satellite galaxies of the Milky Way that emerged after the collision. Linking length distances between $0.1 \mathrm{kpc}$ to $1 \mathrm{kpc}$ were used. A distance of $0.2 \mathrm{kpc}$ suppose that it is compatible with an average distance between stars of $1.4 \mathrm{pc}$ (distance between the sun and Alpha Centauri), but this yielded only two groups, which is not compatible with observations.

The only linking length distance that approximates the number of observed galaxies in the DoS is $1 \mathrm{kpc}$ (Figure 3). For the $12 \%$ mass ratio scenario, 32 groups formed within the tidal tails, whereas 35 groups formed within the tidal tails for the $16 \%$ mass ratio scenario. Although the number of dwarf galaxies in the $12 \%$ mass ratio scenario is compatible with observations, the obtained groups are much closer to the Milky Way center than observations of satellite galaxies show. The mass of these groups is about $1.48 \times 10^{7}$ solar masses, which is compatible with the mass observed for these objects [11].

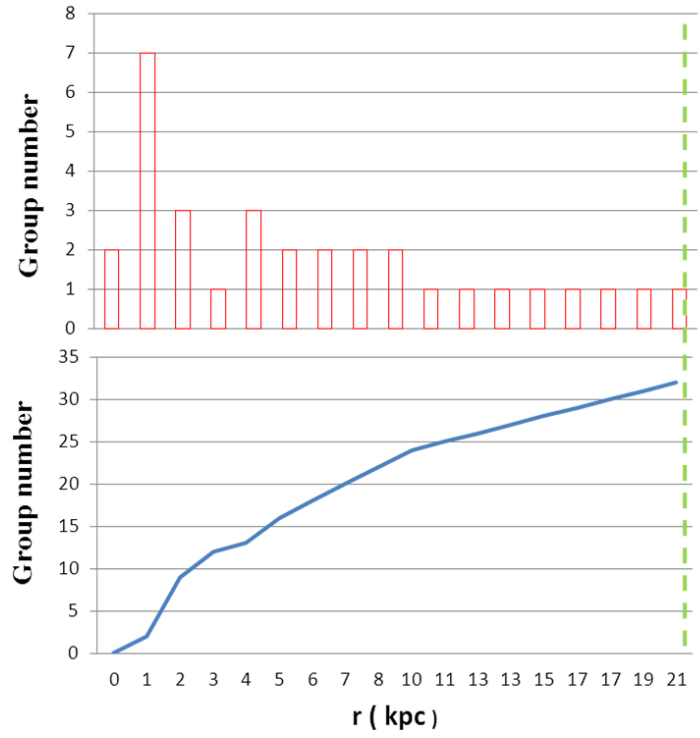

Figures 3 Graph showing the distribution of dwarf satellite galaxies (groups) of the Milky Way due to the collision with the ghost galaxy of $12 \%$ the Milky Way mass. 


\section{Conclusions}

From the results it can be shown that a collision between two disk galaxies can recreate a structure like the DoS. However the characteristics of the structures obtained from the simulations are not similar to those observed.

The number of satellite galaxies found in the collision of $12 \%$ with a range of $1 \mathrm{kpc}$ linking length is compatible with the number of observed galaxies, but the spatial distribution of satellite galaxies after the collision is not consistent with observations.

Regarding the mass of the galaxy incident it can be said that it must be less than $16 \%$ of the Milky Way under the impact parameter of $45 \mathrm{kpc}$, because under these conditions a warming and dismantling of the host galaxy disk is prevented.

\section{References}

[1] Kroupa, C. Theis, and C. M. Boily (2005). The great disk of milky-way satellites and cosmological sub-structures. Astronomy and Astrophysics, 431(2):517-521.

[2] Okazaki, T. and Taniguchi, Y. (2000). Dwarf Galaxy Formation Induced by Galaxy Interactions. ApJ, 543:149-152.

[3] Metz, M., Kroupa, P., and Jerjen, H. (2007). The spatial distribution of the Milky Way and Andromeda satellite galaxies. MNRAS, 374:1125-1145.

[4] Hernquist, L. (1990). An analytical model for spherical galaxies and bulges. ApJ, 356:359-364.

[5] Springel, V. and White, S. D. M. (1999). Tidal tails in cold dark matter cosmologies. MNRAS, 307:162-178.

[6] Toomre, A. and Toomre, J. (1972). Galactic Bridges and Tails. ApJ, 178:623-666. [7] van de Kamp, P. (1964). Elements of Astromechanics. W. H. Freeman and Company.

[8] Pawlowski, M. S., Kroupa, P., and de Boer, K. S. (2011). Making counter-orbiting tidal debris. The origin of the Milky Way disc of satellites? Astronomy and Astrophysics, 532:A118.

[9] Binney. J, T. S. (2008). Galactic Dynamics. Princeton Univ.Press.

[10] McConnachie, A.W. and Irwin, M. J. (2006). The satellite distribution of M31. MNRAS, 365:902-914.

[11] Bullock, J. S., Kaplinghat, M., Fruchter, A., Geha, M., Simon, J., Strigari, L. E., and Willman,B. (2009). Dwarf Galaxies in 2010: Revealing Galaxy Formations Threshold and Testing the Nature of Dark Matter. In astro2010: The Astronomy and Astrophysics Decadal Survey, volume 2010 of ArXiv Astrophysics e-prints, page 32. 